\title{
PERFIL EPIDEMIOLÓGICO DA LEISHMANIOSE VISCERAL NO ESTADO DA BAHIA, BRASIL, NO PERÍODO DE 2014 A 2019
}

\author{
Matheus Teixeira Silva1, Carolina Palmeira Teixeira Martins², Clézio Figueiredo Martins², \\ Alexandre Neves Reis Araújo e Silva ${ }^{3}$, Amanda Rocha Vasconcelos ${ }^{4}$, Dalmir Moreira Santos \\ Filho $^{4}$, Daniel Oliveira Mendes Ferraz ${ }^{4}$, Erickson Gomes de Lima ${ }^{4}$ and \\ Mariane Costa Santos Tavares ${ }^{4}$
}

\begin{abstract}
${ }^{1}$ Autor Correspondente, Discente do Curso de Graduação de Medicina. Faculdade Santo Agostinho - FASA, Vitória da Conquista-Bahia. ${ }^{2}$ Docente do Curso de Graduação de Medicina. Faculdade Santo Agostinho - FASA, Vitória da Conquista-Bahia. ${ }^{3}$ Discente do Curso de Graduação de Medicina. Centro Universitário UNiFG, Guanambi-Ba. ${ }^{4}$ Discente do Curso de Graduação de Medicina. Faculdade Santo
\end{abstract} Agostinho - FASA, Vitória da Conquista-Bahia.

\section{ARTICLE INFO}

\section{Article History:}

Received 04 ${ }^{\text {th }}$ January, 2021

Received in revised form

$19^{\text {th }}$ January, 2021

Accepted $24^{\text {th }}$ February, 2021

Published online $26^{\text {th }}$ March, 2021

\section{Key Words:}

Leishmaniose visceral,

Zoonose, Epidemiologia, Bahia.

*Corresponding author:

Matheus Teixeira Silva

\begin{abstract}
A leishmaniose visceral é uma zoonose causada por espécies do gênero leishmania que pode afetar crianças, jovens, adultos e pessoas imunodeprimidas. A apresentação clínica varia de formas assintomáticas até quadros graves e mortais. No Brasil, é causada pelo protozoário Leishmania infantum chagasi e transmitida por flebotomíneos do gênero Lutzomyia, sendo o cão considerado a principal fonte de infecção no meio urbano. Trata-se de um estudo observacional, descritivo, quantitativobaseado em uma série temporal que empregou dados secundários referentes aoscasos confirmados e notificados de leishmaniose visceral no Estado da Bahia, Brasil,para determinar o perfil epidemiológico no período de 2014 a 2019 . No total foram 2044 casos, acometendo especialmente homens, menores que 5 anos, população de cor parda, moradores de zona urbana e de ensino fundamental incompleto, com maiores incidências nas regiões Centro-Norte, Centro-Leste e Sudoeste. Conclui-se que o Estado da Bahia apresentou elevado número de casos no período analisado e que mais estudos e investigações precisam ser feitos para melhor entendimento e controle da doença.
\end{abstract}

Copyright (C) 2021, Matheus Teixeira Silva et al. This is an open access article distributed under the Creative Commons Attribution License, which permits unrestricted use, distribution, and reproduction in any medium, provided the original work is properly cited.

Citation: Matheus Teixeira Silva, Carolina Palmeira Teixeira Martins, Clézio Figueiredo Martins, Alexandre Neves Reis Araújo e Silva et al.. "Perfil epidemiológico da Leishmaniose Visceral no Estado da Bahia, Brasil, no período de 2014 a 2019”, International Journal of Development Research, 11, (03), 45378-45383.

\section{INTRODUCTION}

A leishmaniose visceral (LV) é uma doença ainda prevalente e que precisa de maior atenção para seu combate. Estima-se que 200 milhões de pessoas estão sobre o risco de infecção pelo protozoário causador da LV. Considerada uma doença endêmica, apresenta $90 \%$ dos casos reportados de seis países: Brasil, Etiópia, Somália, Sudão, Sudão do sul e índia (RODRIGO et al., 2018). O Brasil é responsável por $90 \%$ dos casos registrados na América Latina, com quase três mil pessoas sendo infectadas pela doença anualmente (VON ZUBEN; DONALÍSIO, 2016). A LV é uma doença espectral que pode afetar crianças, jovens, adultos e pessoas imunodeprimidas. A apresentação clínica varia de formas assintomáticas até o quadro clássico de parasitose, evidenciado pela presença de febre, hepatoesplenomegalia e anemia, além de tosse seca, leucopenia, pancitopenia, hipoalbuminemia e hipergamaglobulinemia (SOUZA, et al., 2018). Se não for tratada, esta doença pode se tornar fatal dentro de um ou dois anos após o surgimento da sintomatologia (PRADO et al., 2011). Os agentes etiológicos da LV são protozoários da Família Trypanosomatidae, gênero Leishmania; sendo três espécies que podem causar a doença: Leishmania (Leishmania) donovani na Ásia e África; Leishmania (L.) infantum na Ásia, Europa e África; e Leishmania (L.) infantum chagasi nas Américas (LAINSON; RANGEL, 2005). Os vetores que transmitem a doença são flebotomíneos (Diptera: Psychodidae: Phlebotominae); sendo duas espécies consideradas como vetores no Brasil: Lutzomyia longipalpis e Lutzomyia cruzi, especialmente o primeiro (PRADO et al., 2011). Os cães (Canis familiaris) são identificados como os principais reservatórios doméstico de LV; assim, a detecção de animais infectados é crucial no controle da LV. Mesmo quando eles não apresentam nenhum sintoma clínico da doença, os animais podem ser fonte de parasitas para flebotomíneos que realizam a pastagem sanguínea no animal (PRADO et al., 2011). Visando o combate à doença, o Ministério da Saúde publicou em 2006 o Programa de Vigilância e Controle da Leishmaniose Visceral (PVCLV), cujas 
diretrizes estão centradas na diminuição da morbidade e letalidade, controle do reservatório e dos vetores e na educação em saúde. No entanto alguns problemas ainda são identificados, como a descontinuidade das atividades de controle, resistência dos proprietários de cães com indicação de eutanásia e baixa cobertura do controle químico (VON ZUBEN; DONALÍSIO, 2016). O conhecimento acerca das características epidemiológicas da LV no Estado pode ajudar as autoridades governamentais e as unidades de saúde na elaboração de estratégias para prevenção, controle e tratamento do agravo. Diante disso, este trabalho tem como objetivo entender a situação epidemiológica da leishmaniose visceral no Estado da Bahia, Brasil no período de 2014 a 2019, determinando a incidência geral e por sexo, a letalidade e o perfil sociodemográfico dos pacientes, identificando dificuldades encontradas no seu combate no período avaliado.

\section{MATERIAIS E MÉTODOS}

Trata-se de um estudo observacional, descritivo, quantitativo baseado em uma série temporal que empregou dados secundários referentes aos casos confirmados e notificados de leishmaniose visceral no Estado da Bahia, no período de 2014 a 2019. O Estado da Bahia é uma das 27 unidades federativas do Brasil. Contém 417municípios, sendo Salvador sua capital. Está localizado no sul da região nordeste, sendo o maior estado nordestino com uma área de $564.760,427 \mathrm{~km}^{2}$. Além disso, conta com uma população de 14873064 habitantes, segundo o Instituto Brasileiro de Geografia e Estatística (IBGE), em 2019. Os dados contidos nesse estudo foram obtidos a partir do site DATASUS (www2.datasus.gov.br) na guia Informações em saúde/TABNET, na subseção Epidemiológicas e Morbidades, Doenças e Agravos de Notificação - 2007 em diante, acessado em 20 de novembro de 2020. Foram selecionados os casos de Leishmaniose Visceral notificados no Estado da Bahia no período de 2014 a 2019 através do Sistema de Informação de Agravos de Notificação (SINAN), disponibilizados como domínio público na base de dados do DATASUS. O SINAN é alimentado, principalmente, pela notificação e investigação de casos de doenças e agravos que constam da lista nacional de doenças de notificação compulsória (Portaria de Consolidação $\mathrm{n}^{\circ} 4$, de 28 de Setembro de 2017, anexo V - Capítulo I), mas é facultado a estados e municípios incluir outros problemas de saúde importantes em sua região, como varicela no estado de Minas Gerais ou difilobotríase no município de São Paulo (BRASIL, 2016). Foram analisadas as seguintes variáveis: (1) Número de casos por ano: incidência; (2) Sexo: masculino e feminino; (3) Faixa etária: $<1$, 1-4, 5-19, 20-39, 40- 59, 60-79, 80 ou mais; (4) Raça/Cor da pele: branca, preta, amarela, parda e indígena; (5) Nível de escolaridade: analfabeto, ensino fundamental (completo ou incompleto), ensino médio (completo ou incompleto) e ensino superior (completo ou incompleto); (6) Macrorregião de residência: Centro-Leste, CentroNorte, Extremo Sul, Leste, Nordeste, Norte, Oeste, Sudoeste e Sul; (7) Zona de residência: urbana, rural, periurbana; (8) Evolução: cura, abandono, óbito por LV, óbito por outra causa, transferência. Os dados foram organizados e tabulados no programa computacional Microsoft Excel 2016 para sintetizar e reunir informações de modo estruturado e conciso, facilitando a investigação. As variáveis foram distribuídas em tabelas com os números absolutos e a proporção percentual para cada ano analisado. A incidência foi calculada dividindo o número de casos novos pela população de risco e multiplicando por 100.000 . O coeficiente de letalidade foi calculado dividindo o número de mortespelo total confirmado de casos, multiplicado por 100. A população utilizada como base foi a de 2019, de acordo o IBGE. Por se tratar de uma pesquisa que utilizou dados secundários disponíveis na base de dados do DATASUS, não cabe submissão a Comitê de Ética em Pesquisa (CEP).

\section{RESULTADOS}

No período correspondente de 2014 a 2019, foram notificados no estado da Bahia o total de 2044 casos. Nota-se que 2014 foi o ano em que mais ocorreram notificações de LV, com 524 casos $(25,63 \%)$. O coeficiente de incidência oscilou de 3,18/100.000 hab. a 1,25/100.000 hab., em 2014 e 2019, respectivamente, representando uma diminuição de aproximadamente $60 \%$ (Tabela 1).

Table 1. Número, percentual e coeficiente de incidência dos casos confirmados de Leishmaniose Visceral segundo ano de diagnóstico. Estado da Bahia 2014 - 2019

\begin{tabular}{llll}
\hline $\begin{array}{l}\text { Ano } \\
\text { diagnóstico }\end{array}$ & $\begin{array}{l}\text { Casos } \\
\text { Confirmados } \\
(\mathrm{n})\end{array}$ & $\begin{array}{l}\text { Percentual } \\
(\%)\end{array}$ & $\begin{array}{l}\text { Incidência } \\
(100.000 \text { hab. })\end{array}$ \\
\hline 2014 & 624 & 25,63 & 3,18 \\
2015 & 391 & 19,13 & 2,34 \\
2016 & 247 & 12,08 & 1,47 \\
2017 & 331 & 16,19 & 2,07 \\
2018 & 336 & 16,43 & 2,03 \\
2019 & 215 & 10,52 & 1,25 \\
Total & 2044 & $100 \%$ & \\
\hline Fonte: MS/SVS - Sinan Net. &
\end{tabular}

Tabela 2. Número e distribuição proporcional dos casos confirmados de Leishmaniose Visceral por sexo segundo ano do diagnóstico. Estado da Bahia 2014 - 2019

\begin{tabular}{llllll}
\hline Ano & \multicolumn{4}{l}{ Sexo } & \\
\hline Diagnóstico & \multicolumn{2}{l}{ Masculino } & Feminino \\
& $\mathrm{n}$ & $\%$ & $\mathrm{n}$ & $\%$ & Total \\
2014 & 308 & 58,78 & 216 & 41,22 & 524 \\
2015 & 238 & 60,87 & 153 & 39,13 & 391 \\
2016 & 173 & 70,04 & 74 & 29,96 & 247 \\
2017 & 234 & 70,69 & 97 & 29,3 & 331 \\
2018 & 221 & 65,77 & 115 & 34,23 & 336 \\
2019 & 139 & 64,65 & 76 & 35,34 & 215 \\
Total & 1313 & 64,24 & 731 & 35,76 & 2044 \\
\hline Fonte: MS/SVS - Sinan Net.
\end{tabular}

O número de casos distribuído por sexo foi maior nos homens que representou $64,24 \%$ do total de casos. No total foram 1313 homens e 731 mulheres. Em todo o período os homens foram os mais acometidos, chegando a uma frequência máxima de 70,69\% em 2017 (Tabela 2). Segundo a faixa etária, percebe-se uma distribuição de casos em todos os grupos analisados. Entretanto, a faixa etária mais acometida foi de 1-4 anos, 526 (25,73\%); seguida pela faixa de 5-19 anos, 508 (24,85\%); e 20-39 anos, $437(21,38 \%)$ considerando todo o período. Ocorreram variações em 2015, 2017 e 2019, na qual a faixa etária mais acometida foi de 5-19 anos, com 105 (26,85\%), 82 $(24,77 \%)$ e $57(26,51 \%)$ casos, respectivamente; e em 2016 , a faixa mais acometida foi a de 20-39 anos, com 66 casos (26,72\%) (Tabela 3). Em relação a cor/raça, observa-se um maior número de casos na população parda. Do total, no período analisado, 1384 (67,71\%) eram pardos. O segundo grupo mais acometido foram os pacientes de cor/raça preta, com 301 casos, correspondendo a $14,72 \%$ do total. A cor/raça branca somou $8,17 \%$ do total. Ressalta-se um grande número de ign/branco que foi de 176 noperíodo analisado. As cores/raças indígena e amarela foram as menos acometidas, $0,09 \%$ e $0,86 \%$, respectivamente (Tabela 4).

Em relação à escolaridade, $227(11,1 \%)$ casos tinham a $1^{\mathrm{a}}$ a $4^{\mathrm{a}}$ série incompleta do Ensino Fundamental (EF) e 193 (9,44\%) tinham a 5 a $8^{\mathrm{a}}$ série incompleta do EF. Já quem tinha o ensino médio ou ensino superior completo, o percentual foi de $3,22 \%$ e $0,29 \%$, respectivamente. Importante salientar que houve números elevados nas categorias Ignorado/Branco e Não se aplica, 522 (25,53\%) e 770 $(37,67 \%)$, respectivamente (Tabela 5).

A macrorregião com o maior número de casos foi a região CentroNorte com $428(21,95 \%)$, seguida pela região Centro-lestecom 413 $(21,19 \%)$.A região sudoeste teve um total de 284 casos no período analisado (Tabela 6). De acordo a zona de residência, a urbana aparece com 50,29\% dos casos de LV. 
Tabela 3. Número e distribuição proporcional dos casos confirmados de Leishmaniose Visceral por faixa etária segundo ano do diagnóstico. Estado da Bahia 2014-2019

\begin{tabular}{|c|c|c|c|c|c|c|c|c|c|c|c|c|c|c|c|}
\hline \multicolumn{7}{|l|}{ Ano } & \multicolumn{9}{|c|}{ Faixa Etária } \\
\hline Diagnóstico & $<1$ & & $1-4$ & & $5-19$ & & $20-3$ & & $40-5$ & & $60-79$ & & $80+$ & & Total \\
\hline & $\mathrm{n}$ & $\%$ & $\mathrm{n}$ & $\%$ & $\mathrm{n}$ & $\%$ & $\mathrm{n}$ & $\%$ & $\mathrm{n}$ & $\%$ & $\mathrm{n}$ & $\%$ & $\mathrm{n}$ & $\%$ & \\
\hline 2014 & 37 & 7,06 & 158 & 30,15 & 148 & 28,24 & 90 & 17,17 & 67 & 12,79 & 22 & 4,2 & 2 & 0,38 & 524 \\
\hline 2015 & 31 & 7,93 & 94 & 24,04 & 105 & 26,85 & 78 & 19,95 & 64 & 16,37 & 17 & 4,35 & 2 & 0,51 & 391 \\
\hline 2016 & 12 & 4,86 & 55 & 22,27 & 53 & 21,46 & 66 & 26,72 & 40 & 16,19 & 21 & 8,5 & 0 & 0 & 247 \\
\hline 2017 & 24 & 7,25 & 70 & 21,15 & 82 & 24,77 & 72 & 21,75 & 63 & 19,03 & 18 & 5,44 & 2 & 0,6 & 331 \\
\hline 2018 & 17 & 5,06 & 100 & 29,76 & 63 & 18,75 & 76 & 22,62 & 52 & 15,48 & 23 & 6,84 & 5 & 1,49 & 336 \\
\hline 2019 & 7 & 3,25 & 49 & 22,79 & 57 & 26,51 & 55 & 25,58 & 31 & 14,42 & 15 & 6,97 & 1 & 0,46 & 215 \\
\hline Total & 128 & 6,26 & 526 & 25,73 & 508 & 24,85 & 437 & 21,38 & 317 & 15,5 & 116 & 5,67 & 12 & 0,58 & 2044 \\
\hline
\end{tabular}

Fonte: MS/SVS -Sinan Net.

Tabela 4. Número e distribuição proporcional de casos confirmados de Leishmaniose Visceral por cor/raça segundo ano do diagnóstico. Estado da Bahia 2014-2019

\begin{tabular}{|c|c|c|c|c|c|c|c|c|c|c|c|c|c|}
\hline \multirow[t]{2}{*}{ Ano Diagnóstico } & \multicolumn{2}{|c|}{ Ign/Branco } & \multicolumn{2}{|c|}{ Branca } & \multicolumn{2}{|c|}{ Preta } & \multicolumn{2}{|l|}{ Amarela } & \multicolumn{2}{|l|}{ Parda } & \multicolumn{3}{|l|}{ Indígena } \\
\hline & $\mathrm{n}$ & $\%$ & $\mathrm{n}$ & $\%$ & $\mathrm{n}$ & $\%$ & $\mathrm{n}$ & $\%$ & $\mathrm{n}$ & $\%$ & $\mathrm{n}$ & $\%$ & Total \\
\hline 2014 & 44 & 8,4 & 54 & 10,4 & 76 & 14,5 & 3 & 0,57 & 347 & 66,22 & 0 & 0 & 524 \\
\hline 2015 & 21 & 5,37 & 30 & 7,67 & 55 & 14,07 & 1 & 0,25 & 283 & 72,38 & 1 & 0,25 & 391 \\
\hline 2016 & 32 & 12,95 & 28 & 11,34 & 34 & 13,76 & 1 & 0,4 & 152 & 61,54 & 0 & 0 & 247 \\
\hline 2017 & 26 & 7,85 & 24 & 7,25 & 44 & 13,29 & 3 & 0,91 & 234 & 70,69 & 0 & 0 & 331 \\
\hline 2018 & 31 & 9,23 & 24 & 7,14 & 60 & 17,86 & 3 & 0,89 & 217 & 64,58 & 1 & 0,3 & 336 \\
\hline 2019 & 22 & 10,23 & 7 & 3,25 & 32 & 14,88 & 3 & 1,39 & 151 & 70,23 & 0 & 0 & 215 \\
\hline Total & 176 & 8,61 & 167 & 8,17 & 301 & 14,72 & 14 & 0,86 & 1384 & 67,71 & 2 & 0,09 & 2044 \\
\hline
\end{tabular}

Fonte: MS/SVS -Sinan Net

Tabela 5. Número e percentual de casos confirmados de Leishmaniose Visceral por escolaridade segundo ano do diagnostic Estado da Bahia 2014 - 2019

\begin{tabular}{|c|c|c|c|c|c|c|c|c|c|c|c|c|}
\hline \multirow[t]{2}{*}{$\begin{array}{l}\text { Ano } \\
\text { diagnóstico }\end{array}$} & \multicolumn{2}{|c|}{ Ign/Branco } & \multicolumn{2}{|c|}{ Analfabeto } & \multicolumn{2}{|c|}{$\begin{array}{l}1^{\mathrm{a}} \text { a } 4^{\mathrm{a}} \text { série } \\
\text { Incomplete do EF }\end{array}$} & \multicolumn{2}{|c|}{$\begin{array}{l}4^{\mathrm{a}} \text { série } \\
\text { Complete do EF }\end{array}$} & \multicolumn{2}{|c|}{$\begin{array}{l}5 \text { a } 8^{\mathrm{a}} \text { série } \\
\text { Incomplete do EF }\end{array}$} & \multicolumn{2}{|c|}{ EF completo } \\
\hline & $\mathrm{n}$ & $\%$ & $\mathrm{n}$ & $\%$ & $\mathrm{n}$ & $\%$ & $\mathrm{n}$ & $\%$ & $\mathrm{n}$ & $\%$ & $\mathrm{n}$ & $\%$ \\
\hline 2014 & 107 & 20,42 & 15 & 2,86 & 65 & 10,4 & 22 & 4,2 & 41 & 7,82 & 10 & 1,91 \\
\hline 2015 & 83 & 22,23 & 13 & 3,32 & 52 & 13,3 & 17 & 4,35 & 40 & 10,23 & 10 & 5,56 \\
\hline 2016 & 71 & 28,74 & 6 & 2,43 & 19 & 7,69 & 14 & 5,67 & 27 & 10,93 & 7 & 2,83 \\
\hline 2017 & 104 & 31,42 & 11 & 3,32 & 45 & 13,59 & 16 & 4,83 & 30 & 9,06 & 3 & 0,91 \\
\hline 2018 & 94 & 27,98 & 8 & 2,38 & 29 & 8,63 & 12 & 3,57 & 21 & 6,25 & 4 & 1,19 \\
\hline 2019 & 63 & 29,3 & 12 & 5,58 & 17 & 7,9 & 6 & 2,79 & 24 & 11,16 & 7 & 3,25 \\
\hline Total & 522 & 25,53 & 65 & 3,18 & 227 & 11,1 & 87 & 4,25 & 193 & 9,44 & 41 & 2 \\
\hline
\end{tabular}

Em todos os anos, a zona urbana aparece com mais casos que a zona rural, exceto no ano de 2017 em que há um percentual maior na zona rural (Tabela 7). Na evolução dos casos, observa-se $1257(61,49 \%)$ curas e $136(6,65 \%)$ óbitos no período analisado. Destaca-se também o elevado número de ignorado/Branco que foi de 463 (22,65\%), no qual não é possível determinar se esses pacientes evoluíram para a cura ou óbito. A taxa de letalidade oscilou de 5,78\% a 8,93\% em 2014 e 2018, respectivamente (Tabela 8).

\section{DISCUSSÃO}

Aproximadamente 200.000 casos de LV foram reportados à Organização Mundial da Saúde (OMS) em 2015. Entre as doenças tropicais, as leishmanioses são a segunda causa de maior mortalidade e a sétima em perda de anos de vida ajustados por incapacidade (BERNY, 2020). No Brasil, a doença continua em franca expansão. A doença deixa de ter caráter rural e passa a se expandir e se tornar de caráter urbano no país a partir da década de 1980, com o registro de transmissão em áreas urbanizadas em cidades de maior dimensão, sendo os cães o principal hospedeiro do reservatório (LUZ et al., 2019). Anteriormente restrita ao Nordeste, dissemina-se por várias cidades do Norte, Centro-oeste e Sudeste, causando epidemias urbanas e aumento de incidência e letalidade (VON ZUBEN; DONALÍSIO, 2016). No Estado da Bahia, Brasil, no período de 2014 a 2019, 2044 casos foram notificados, com média de 340,6 casos por ano, apresentando uma tendência temporal de regressão, com 524 casos notificados em 2014, ano de maior notificação, e 215 em 2019, ano com menor número de notificação. $\mathrm{O}$ coeficiente de incidência reduziu de 3,18 em 2014 para 1,25 no ano de 2019.
Essa diminuição pode demonstrar que as medidas de prevenção da doença, principalmente contra o vetor, têm mostrado resultados satisfatórios. No entanto, esses números podem ser mais elevados ao considerar subnotificação de casos e a demora entre o início dos sintomas e o diagnóstico clínico. Analisando a variável sexo, observase que em todos os anos, a população masculina é a mais acometida: $64,24 \%$ dos acometidos no período são homens. Esse resultado também foi encontrado em outros trabalhos como o realizado no Estado do Ceará entre os anos 2003-2017 (CAVALCANTE et al., 2020). A maior prevalência nos homens pode ser justificada pela maior exposição aos vetores flebotomíneos e não por maior suscetibilidade (OLIVEIRA; PIMENTA, 2014). Em relação a faixa etária, todas as idades foram acometidas pela doença. Entretanto, três grupos foram mais prevalentes: 1 a 4 anos, 5 a 19 anos e 20 a 39 anos. Destaca-se que $50,58 \%$ dos casos pertenceram a faixa etária de 1-19 anos. No estudo realizado no Estado de Alagoas entre os anos de 2007 a 2013 também mostrou maior distribuição dos casos em crianças e adultos (ROCHA et al., 2018). A ocorrência da doença em adultos pode ser justificada por sua maior exposição aos flebotomíneos vetores, por serem considerados pertencentes ao grupo dos indivíduos economicamente ativos (SOUZA et al., 2018). Já em crianças de 1 a 4 anos, acredita-se que o maior risco de carência nutricional, condição comum nas áreas endêmicas, e o estado imunológico ainda em formação possa justificar essa maior prevalência (ORTIZ; ANVERSA, 2015). Segundo a cor/raça, a parda foi a de maior ocorrência. Esse resultado contrasta o estudo realizado em Sobral, São Paulo, no período de 2004 a 2012, no qual 49,3\% dos casos foram de cor/raça branca. Essa diferença pode ser explicada pela maior frequência da cor/raça parda na população baiana, onde a população é composta principalmente 
Tabela 6. Número e percentual de casos confirmados de Leishmaniose Visceral por macrorregião de Saúde de residência segundo ano de diagnóstico. Estado da Bahia 2014-2019

\begin{tabular}{|c|c|c|c|c|c|c|c|c|c|c|c|c|c|c|c|c|c|c|c|}
\hline Ano notificação & Sul & & Sudoeste & & Oeste & & Norte & & Nordeste & & Leste & & & emo Sul & Centr & o-leste & Cent & - Norte & \\
\hline & $\mathrm{n}$ & $\%$ & $\mathrm{n}$ & $\%$ & $\mathrm{n}$ & $\%$ & $\mathrm{n}$ & $\%$ & $\bar{n}$ & $\%$ & $\mathrm{n}$ & $\%$ & $\bar{n}$ & $\%$ & $\mathrm{n}$ & $\%$ & $\bar{n}$ & $\%$ & Total \\
\hline 2014 & 15 & 2,97 & 67 & 13,26 & 43 & 8,51 & 42 & 8,31 & 9 & 1,78 & 83 & 16,43 & 1 & 0,19 & 140 & 27,72 & 105 & 20,79 & 505 \\
\hline 2015 & 19 & 5,09 & 54 & 14,47 & 48 & 12,86 & 33 & 8,84 & 1 & 0,26 & 42 & 11,26 & - & 0 & 66 & 17,69 & 110 & 29,49 & 373 \\
\hline 2016 & 9 & 3,91 & 47 & 20,43 & 30 & 13,04 & 11 & 4,78 & 7 & 3,04 & 41 & 17,82 & 1 & 0,43 & 31 & 13,47 & 53 & 23,04 & 230 \\
\hline 2017 & 16 & 5,07 & 51 & 16,19 & 57 & 18,09 & 23 & 7,3 & 2 & 0,63 & 49 & 15,55 & 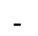 & 0 & 49 & 15,55 & 68 & 21,58 & 315 \\
\hline 2018 & 11 & 3,47 & 42 & 13,24 & 55 & 17,35 & 17 & 5,36 & 3 & 0,94 & 66 & 20,82 & - & 0 & 71 & 22,39 & 52 & 16,4 & 317 \\
\hline 2019 & 4 & 1,91 & 23 & 11 & 25 & 11,96 & 12 & 5,74 & 1 & 0,48 & 47 & 22,48 & 1 & 0,48 & 56 & 26,79 & 40 & 19,13 & 209 \\
\hline Total & 74 & 3,79 & 284 & 14,57 & 258 & 13,23 & 138 & 7,08 & 23 & 1,18 & 328 & 16,82 & 3 & 0,15 & 413 & 21,19 & 428 & 21,95 & 1949 \\
\hline
\end{tabular}

Fonte: MS/SVS - Sinan Net.

Tabela 7. Número e percentual de casos de Leishmaniose Visceral por zona de residência segundo ano diagnóstico.Estado da Bahia $2014-2019$

\begin{tabular}{llllllllll}
\hline Ano Notificação & Ign/Branco & & Urbana & & Rural & & Periurbana & Total \\
\hline & $\mathrm{n}$ & $\%$ & $\mathrm{n}$ & $\%$ & $\mathrm{n}$ & $\%$ & $\mathrm{n}$ & $\%$ & $\mathrm{n}$ \\
2014 & 16 & 3,05 & 272 & 51,9 & 233 & 44,46 & 3 & 0,57 & 524 \\
2015 & 9 & 2,3 & 192 & 49,1 & 187 & 47,82 & 3 & 0,76 & 391 \\
2016 & 5 & 2,02 & 139 & 56,27 & 101 & 40,89 & 2 & 0,8 & 247 \\
2017 & 12 & 3,62 & 153 & 46,22 & 162 & 48,94 & 4 & 1,2 & 331 \\
2018 & 16 & 4,76 & 161 & 47,91 & 154 & 45,83 & 5 & 1,48 & 336 \\
2019 & 10 & 4,65 & 111 & 51,62 & 92 & 42,79 & 2 & 0,93 & 215 \\
Total & 68 & 3,32 & 1028 & 50,29 & 929 & 45,45 & 19 & 0,92 & 2044 \\
\hline
\end{tabular}

Fonte: MS/SVS - Sinan Net.

Tabela 8. Número e percentual de casos de Leishmaniose Visceral por evolução segundo ano diagnóstico. Estado da Bahia $2014-2019$

\begin{tabular}{|c|c|c|c|c|c|c|c|c|c|c|c|c|c|}
\hline \multirow{2}{*}{ Evolução } & \multicolumn{2}{|c|}{ Ign/Branco } & \multicolumn{2}{|l|}{ Cura } & \multicolumn{2}{|c|}{ Abandono } & \multicolumn{2}{|c|}{ Óbito por LV } & \multicolumn{2}{|c|}{ Óbito por outra causa } & \multicolumn{2}{|c|}{ Transferência } & \multirow[b]{2}{*}{ Total } \\
\hline & $\mathrm{n}$ & $\%$ & $\mathrm{n}$ & $\%$ & $\mathrm{n}$ & $\%$ & $\mathrm{n}$ & $\%$ & $\mathrm{n}$ & $\%$ & $\mathrm{n}$ & $\%$ & \\
\hline 2014 & 101 & 18,23 & 329 & 62,79 & 3 & 0,54 & 32 & 5,78 & 8 & 1,44 & 51 & 9,2 & 554 \\
\hline 2015 & 99 & 25,32 & 244 & 62,4 & 5 & 1,28 & 24 & 6,14 & 5 & 1,28 & 14 & 3,58 & 391 \\
\hline 2016 & 81 & 32,79 & 129 & 52,23 & 3 & 1,21 & 14 & 5,67 & 7 & 2,83 & 13 & 5,26 & 247 \\
\hline 2017 & 61 & 18,43 & 216 & 65,26 & 2 & 0,6 & 22 & 6,65 & 7 & 2,11 & 18 & 5,44 & 331 \\
\hline 2018 & 59 & 17,56 & 215 & 63,99 & 3 & 0,89 & 30 & 8,93 & 5 & 1,49 & 24 & 7,14 & 336 \\
\hline 2019 & 62 & 28,83 & 124 & 57,67 & 0 & 0 & 14 & 6,51 & 2 & 0,93 & 13 & 6,04 & 215 \\
\hline Total & 463 & 22,65 & 1257 & 61,49 & 16 & 0,78 & 136 & 6,65 & 39 & 1,9 & 133 & 6,5 & 2044 \\
\hline
\end{tabular}

Fonte: MS/SVS - Sinan Net. 
por autodeclarados pardos. No último Censo demográfico de 2010, aproximadamente $60 \%$ da população da Bahia eram da cor/raça parda (IBGE, 2010). No que diz respeito à escolaridade, verifica-se que o número de indivíduos acometidos com alta escolaridade (Ensino médio completo e Ensino Superior completo) é baixo (3,65\% do total). Provavelmente, isso se deve ao maior acesso às informações sobre medidas preventivas. Já os indivíduos com Ensino Fundamental incompleto $\left(1^{\mathrm{a}}\right.$ a $4^{\mathrm{a}}$ série incompleta do EF e $5^{\mathrm{a}}$ a $8^{\mathrm{a}}$ série incompleta do EF) compõem uma parte significativa de doentes (20,54\%), evidenciando que a alta prevalência da LV nesse grupo pode estar associada a menor educação em saúde. No entanto, os graus de instrução ignorados/brancos e os que não se aplicam, juntos correspondem a $63,2 \%$ do total, podendo ser justificado, em parte, pelo fato de que $32 \%$ dos casos correspondem a menores de 4 anos, os quais ainda não frequentam o ambiente escolar. No estudo realizado em Sobral, no período de 2011 a 2015,foram obtidos resultados semelhantes (SOUZA et al., 2018).

Dentre as macrorregiões de residência, a maior porcentagem se encontrava na macrorregião Centro-Norte (21,95\%), local onde se localiza as Cidades deJacobina e Irecê, localidades de importante crescimento urbano e agrícola. A região Centro-Leste, na qual se localiza a cidade de feira de Santana, ficou com proporções semelhantes, $21,19 \%$. Esta região é a segunda mais populosa da Bahia e experimentou recentemente um rápido crescimento populacional devido a um intenso fluxo migratório de outras regiões do Brasil, o que tem sido atribuído à industrialização e ao avanço da agricultura local. Isso pode ajudar, a explicar em parte essa maior prevalência nesses locais. No entanto, outros fatores como ambiente, clima, quantidade de reservatórios também são importantes. Destaca-se a possibilidade de subnotificação em outras regiões que poderiam gerar resultados diferentes desse trabalho. Segundo a zona de residência, observa-se que houve uma prevalência da zona urbana em relação à zona rural ao longo dos anos. Esse fato está diretamente relacionado com a rápida transição demográfica associada ao aumento da urbanização e a adaptação do vetor ao peridomicílio (CAVALCANTE et al., 2020), bem como a disseminação de reservatórios infectados a áreas sem transmissão e às mudanças climáticas e ambientais (MAIA-ELKHOURY et al., 2008). Na evolução dos indivíduos, $1257(61,49 \%)$ obtiveram a cura como desfecho. Entretanto a taxa de letalidade variou de 5,67 a 8,93 com um total de 136 óbitos no período avaliado. Esses resultados mostram uma boa taxa de cura, o que pode ser explicado pelo maior acesso aos serviços de saúde e tratamento. No entanto, ainda há um número significativo de mortes, além de possíveis morbidades que não foram avaliadas nesse trabalho.

A letalidade média no período foi de $6,65 \%$ sendo essa maior que a média nacional de $5,7 \%$ (BRASIL, 2011) e menor que a taxa das américas que foi de 8\% (OMS, 2019). O Programa de Vigilância e Controle de Leishmaniose Visceral tem priorizado esforços para prevenir aproximadamente 250 mortes anuais em decorrência da grande viabilidade de se conseguir esse resultado, comparado a uma substancial redução na incidência (WERNECK, 2016). Dos 2044 casos, $83(4 \%)$ foram recidivas. Isso pode ser explicado por ineficácia do tratamento, abandono e diminuição da capacidade de resposta do sistema imunológico da pessoa doente, além de comorbidades associadas a LV (SOUZA et al., 2018). A redução da letalidade por LV requer melhora na estruturação das unidades de saúde, bem como a capacitação e atualização dos profissionais de saúde para o reconhecimento precoce dos sinais e sintomas clínicos e, assim, promover o manejo clínico-terapêutico adequado dos pacientes. Luz et al (2019) identificou que vários pacientes procuram atendimento público em Unidades Básicas de Saúde e unidades de emergências no início dos sintomas da LV. No entanto, a maioria teve que visitar várias unidades de saúde adicionais antes de surgir uma suspeita clínica e um diagnóstico correto ser feito. Constata-se, dessa forma, uma fragilidade da atenção primária à saúde em reconhecer prontamente a LV e isso pode ser atribuído aos sintomas clínicos inespecíficos apresentados pelos pacientes, que geralmente se sobrepõem aos de outros síndromes febris, carência de profissionais de saúde capacitados, além de limitações tanto na disponibilidade e viabilidade de recursos e métodos laboratoriais em estabelecimentos de atenção primária (BARBOSA et al., 2013). Esse estudo apresentou limitações por utilizar dados secundários, susceptíveis a erros de preenchimento, o que pode ter acarretado em algum grau de quantidade de informações ignoradas e vieses de informações distintos. Tendo em vista tal ótica, sugere-se que mais estudos epidemiológicos sejam realizados, principalmente em áreas de risco, além de melhorias nas políticas públicas e conscientização da população.

\section{CONCLUSÕES}

O presente estudo evidenciou uma média de 340,6 casos por ano de Leishmaniose Visceral no Estado da Bahia, acometendo especialmente homens, menores que 5 anos, população de cor parda, moradores de zona urbana e de ensino fundamental incompleto, com maiores incidências nas regiões Centro-Norte, Centro-Leste e Sudoeste, respectivamente. Diante disso, constata-se que é de extrema importância o conhecimento acerca da LV, bem como da sua epidemiologia para a suspeita clínica em pacientes com sintomas inespecíficos ou com febre e hepatoesplenomegalia tendo o perfil epidemiológico como um dos fatores contribuintes para o raciocínio diagnóstico. O conhecimento epidemiológico também permite estimular a implementação de políticas públicas para ampliar os recursos humanos e materiais disponíveis com o intuito de oferecer melhores condições para diagnosticar e tratar precocemente os casos de LV, objetivando, assim, a redução da incidência e da mortalidade da doença.

\section{REFERÊNCIAS}

BARBOSA, M.N., et al. Atenção aos casos humanos de leishmaniose visceral no âmbito da atenção primária à saúde em município da região metropolitana de belo horizonte. Rev. APS. v. 16 n. 3, p. 234-241, jul/set, 2013.

BERN, Caryn. Visceral leishmaniasis: Epidemiology and control. UpToDate, 2020. Disponível em <https://www.uptodate.com>. Acesso em: 14/03/2020.

BRASIL. Ministério da Saúde. Secretaria de Vigilância em saúde. Departamento de Vigilância Epidemiológica. Leishmaniose Visceral: recomendações clínicas para redução da letalidade. Brasília: Ministério da Saúde; 2011.

BRASIL. Ministério da Saúde. O Sinan. 2016. Disponível em $<$ http://www.portalsinan.saude.gov.br/o-sinan>. Acesso em 01/03/2021

CAVALCANTE, F. R. A., et al. Human Visceral leishmaniasis: epidemiological, temporal and spacial aspects in Northeast Brazil, 2003-2017. Rev Inst Med Trop, São Paulo, v. 62, n. 12, 2020.

IBGE. SIDRA. Censo demográfico, Tabela 3175 - População residente, por cor ou raça, segundo a situação do domicílio, o sexo e a idade. Disponível em <https://sidra.ibge.gov.br/ Tabela/3175>. Acesso em 01/03/2021.

LAINSON, R; RANGEL, E.F. Lutzomyia longipalpis and the ecoepidemiology of American visceral leishmaniasis, with particular reference to Brazil - A Review. Mem Inst Oswaldo Cruz, Rio de Janeiro, v. 100, n. 8, p. 811-827, dez., 2005.

LUZ, J. G. G., et al. Where, when, and how the diagnosis of human visceral leishmaniasis is defined: answers from the Brazilian control program. Mem Inst Oswaldo Cruz, Rio de Janeiro, v. 114, 2019.

MAIA-ELKHOURY, A. N., et al. Visceral leishmaniasis in Brazil: trends and challenges. Cad Saude Publica, v. 24, 2008.

OLIVEIRA, E.N; PIMENTA, A.M. Perfil epidemiológico da leishmaniose visceral no município de Paracatu, MG no período de 2007 a 2010. REME Rev. Min. Enferm., v. 18, n. 2, p. 365375 .

Organização Mundial da Saúde. Organização Pan-Americana de Saúde. Leishmanioses: Informe Epidemiológico das Américas. Informe de Leishmanioses, n. 8, dezembro, 2019. 
ORTIZ, R. C., ANVERSA, L. Epidemiologia da leishmaniose visceral em Bauru, São Paulo, no período de 2004 a 2012: um estudo descritivo. Epidemiol Serv Saude, v. 24, p. 97-104, 2015.

PRADO, P.F. et al. Epidemiological aspects of human and canine visceral leishmaniasis in Montes Claros, State of Minas Gerais, Brazil, between 2007 and 2009. Revista da Sociedade Brasileira de Medicina Tropical, v. 44, n. 5, p. 561-566, set-out, 2011.

ROCHA, M. A. N., et al. Epidemiological aspects of human and canine visceral leishmaniasis in State of Alagoas, Northeast, Brazil. Braz. J. Biol., v. 78, n. 4, p. 609-614, 2018.
RODRIGO, C; et al. Amphotericin B for treatment of visceral leishmaniasis: systematic review and meta-analysis of prospective comparative clinical studies including dose-ranging studies. Clinical Microbiology and Infection, v. 24, p. 591-598, 2018.

SOUSA, Natanael Aguiar de; et al. Perfil epidemiológico dos casos de leishmaniose visceral em Sobral-Ce de 2011 a 2015. SANARE, Sobral, v.17, n.01, p.51-57, jan./jun. 2018.

VON ZUBEN, A. P. B., DONALÍSIO, M. R. Dificuldades na execução das diretrizes do Programa de Vigilância e Controle da Leishmaniose Visceral em grandes municípios brasileiros. Cad. Saúde Pública, Rio de Janeiro, v. 32, n. 6, jun., 2016.

WERNECK, G. L. The control of visceral leishmaniasis in Brazil: end of a cycle? Cad. Saúde Pública, Rio de Janeiro, v. 32, 2016. 